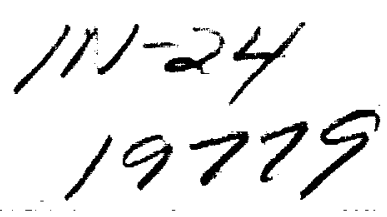

NASA Technical Memorandum 104464

AIAA-91-1739

$\beta \cdot \beta$

\title{
Increased Heat Transfer to a Cylindrical Leading Edge due to Spanwise Variations in the Freestream Velocity
}

D.L. Rigby

Sverdrup Technology, Inc.

Lewis Research Center Group

Brook Park, Ohio

and

G.J. Van Fossen

National Aeronautics and Space Administration

Lewis Research Center

Cleveland, Ohio

Prepared for the

22nd Fluid Dynamics, Plasma Dynamics and Lasers Conference sponsored by the American Institute of Aeronautics and Astronautics Honolulu, Hawaii, June 24-26, 1991

\section{N/SA}

(NASA-TM-104464) INCREASEO HEAT TRANSFFR TD

A CYLINRRICAL LEATING EDGE DUE TO SPANWISE

VARIATIONS IN THE FREESTREAM VELUCITY

(NASA) $13 \mathrm{P}$

CSCL 110 


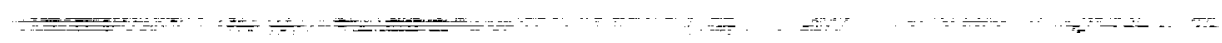




\title{
INCREASED HEAT TRANSFER TO A CYLINDRICAL LEADING EDGE DUE TO SPANWISE VARIATIONS IN THE FREESTREAM VELOCITY
}

\author{
D. L. Rigby* \\ Sverdrup Technology Inc. \\ NASA Lewis Research Group \\ Brookpark, Ohio \\ and \\ G. J. Van Fossen \\ NASA Lewis Research Center \\ Cleveland, Ohio
}

\begin{abstract}
The present study numerically demonstrates how small spanwise variations in velocity upstream of a body can cause relatively large increases in the spanwise-averaged heat transfer to the leading edge. Vorticity introduced by spanwise variations, first decays as it drifts downstream, then amplifies in the stagnation region as a result of vortex stretching. This amplification can cause a periodic array of threedimensional structures, similar to horseshoe vortices, to form. The numerical results indicate that, for a given wavelength, there is an amplitude threshold below which a structure does not form. A onedimensional analysis, to predict the decay of vorticity in the absence of the body, in conjunction with the full numerical results indicates that the threshold is more accurately stated as a minimum level of vorticity required in the leading edge region for a structure to form. It is possible, using the one-dimensional analysis, to compute an optimum wavelength in terms of the maximum vorticity reaching the leading edge region for given amplitude. A discussion is presented which relates experimentally observed trends to the trends of the present phenomena.
\end{abstract}

\section{Nomenclature}

A peak to peak velocity difference normalized by the average velocity

$d$ diameter of wires in screen

$\mathrm{Fr} \quad$ Frossling number $(\mathrm{Fr}=\mathrm{Nu} / \sqrt{R e})$

$k$ thermal conductivity

$M \quad$ Mach number

$\mathrm{Nu} \quad$ Nusselt number

$p \quad$ pressure

$q_{w} \quad$ wall heat transfer

$R \quad$ leading edge radius

$R e_{x} \quad$ Reynolds number (i.e., $R e_{x}=\rho_{0} \bar{U} x / \mu_{0}$ )

$T$. temperature

$u \quad$ streamwise velocity

$v \quad$ normal velocity

* Member AIAA

$\begin{array}{ll}w & \text { spanwise velocity } \\ u_{1} & \left.\text { velocity difference (ie. } u_{1}=u-\bar{U}\right) \\ u_{\min } & \text { minimum streamwise velocity in } \\ & x-z \text { symmetry plane } \\ \bar{U} & \text { spanwise-averaged freestream velocity } \\ x & \text { streamwise direction } \\ \triangle x & \text { distance from } x_{0} \\ x_{w i r e} & \text { denotes position of screen } \\ y & \text { normal direction } \\ z & \text { spanwise direction }\end{array}$

Greek Symbols

$\lambda \quad$ wavelength of spanwise variation

$\gamma \quad$ ratio of specific heats

$\mu \quad$ viscosity of fluid

$\Omega \quad$ vorticity oriented in the $y$-direction

$\tilde{\Omega} \quad$ normalized vorticity predicted by the analysis (ie. $\tilde{\Omega}=\Omega /(\pi \bar{U} / R)$ )

density of fluid

solidity of array of wires

distance along surface normalized by the leading edge radius

\section{Subscripts and Superscripts}

() conditions at the exit

() f represents the mean value in the boundary layer (ie. film)

() $L . E$ denotes leading edge

() $\min$ represents the minimum value on the stagnation line

()$_{\max } \quad$ represents the maximum value on the stagnation line

() wavelength for which analysis predicts maximum peak vorticity at leading edge

() $t \quad$ total conditions

() th threshold point at which structure forms

()$_{w} \quad$ value at wall

() wire denotes position of array of wires

() denotes upstream boundary

() spanwise-averaged quantity 


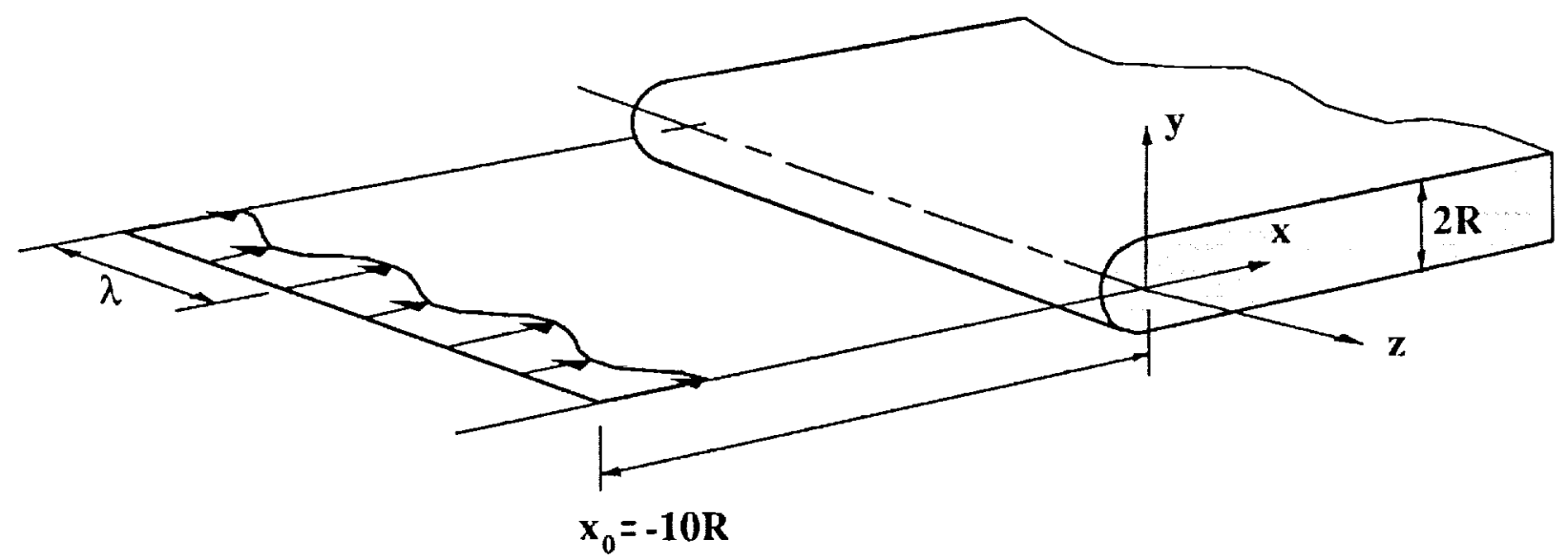

Figure 1: Geometry of Problem

\section{Introduction}

The prediction of heat transfer to the leading edge of gas turbine blades is very important to the design process. Typically, the leading edge is a location of high heat transfer. It has been known for some time that relatively small increases in freestream turbulence above a nominally laminar flow can cause large increases in the heat transfer at a forward stagnation region [1]. However, the mechanism by which such an increase occurs is not well understood. It has also been observed experimentally that spanwise variations in the heat transfer occur, with wavelengths that vary inversely as the square root of the Reynolds number, in flows with very small spanwise variations in the freestrean velocity. This phenomena has becn studied experimentally as it influences heat transfer $[2,3]$ and mass transfer $[4,5,6]$. The experiments indicate that spanwise velocity variations on the order of $1 \%$ can cause the transfer coefficients to increase on the order of $20 \%$.

Asymptotic analysis for small perturbations in the spanwise direction $[7,8,9,10,11]$ indicate that disturbances with a wavelength greater than some neutral wavelength can be amplified in the boundary layer. However, prediction of the neutral wavelength and the most unstable wavelength differs from author to author. In fact, Iammerlin [12] found instability for a continuous spectrum of wave numbers, while Wilson and Gladwell [13] found the flow was always stable to infinitesimal disturbances. More recently, Thompson [14] found that stagnation flow exhibits instability if unsteadiness is introduced into the basic state of the flow. Itis analysis suggests that there is an amplitude threshold on the unsteadiness for instability to occur.

In a review of this type of flow, Morkovin [15] discusses the limits of the available analysis tools as well as the uncertainties in experiments. It is unclear whether the major effects are a result of the random unsteady turbulence or a result of large, essentially steady, three-dimensional structures. These struct ures could be the result of small spanwise perturbations in the freestream. Morkovin [15] also raises thic question of forced or free response: "The general impressions seem to be that some forcing is present and that somehow the characteristics of the boundary layer on the cylinder enter the quantitative detemination of the geometry of the horseshoc vortices." Based on a combination of flow visualization, turbulence measurements, and spanwise heat transfer measurements, Vau Fossen and Simoneau [2] arrived at the same general impressions.

Numerical studies attempting to model the turbulence $[16,17,18]$ have also been undertaken with a certain degree of success. However, if the experimental results are dominated by a steady spatial variation or even a nonrandom variation in the third dimension, a two-dimensional calculation with a turbulence model will not properly predict the flow features.

The present work will concentrate on the effect of a steady spanwise velocity variation on the heat transfer at the leading edge. Numerical solutions of the [ull three dimensional Navier-Stokes equations are used to investigate the requircments for the formation of a horseshoc-like vortex, and to show the effect of these vortices on the heat transfer coeflicient at the leading edge. In addition, a parallel flow analysis which predicts the decay of vorticity in the absence of the body is cmployed. This analysis proves to be useful in predicting the threshold level of vorticity for the formation of a vortex structure. The parallel flow analysis also predicts the optimum wavelength in terms of supplying the maximum vorticity to the leading edge region.

\section{Statement of Problem}

Consider the problem of a flat plate with a cylindrical leading edge of radius $R$, Figure 1 . The body is assumed to be infinite and uniform in the spanwise 


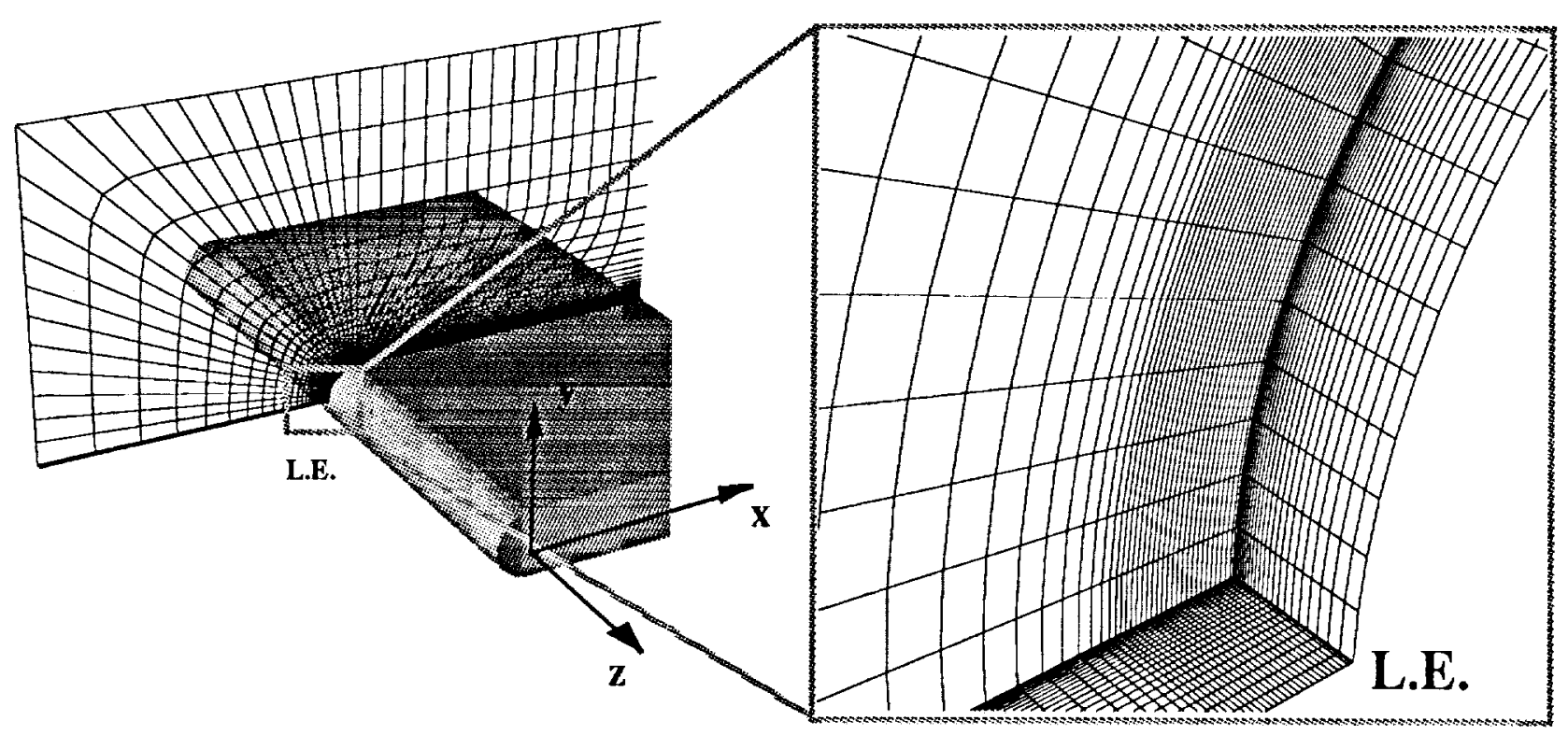

Figure 2: $37 \times 51 \times 22$ grid for $\lambda=0.4 R$

direction, and semi-infinite in the streamwise direction. Upstream of the body, the flow has a sinusoidal variation of velocity in the spanwise direction with a wavelength $\lambda$, which introduces vorticity oriented perpendicular to the $x-z$ plane. The heat flux on the body is fixed at a uniform value. The peak to peak velocity variation as a percentage of the average upstream velocity is defined as $A_{0}$ and the wavelength is denoted by $\lambda$. The velocity distribution is imposed at a position upstream of the leading edge, denoted by $x_{0}$. This position is very important to note since the vorticity first decays as it approaches the body, then is amplified in the region near the leading edge. By ignoring the presence of the body, the rate of decay of the vorticity can be estimated from a parallel flow analysis, as will be shown below.

\section{Solution Method}

The solution was computed using the PARC3D code, which uses the Beam and Warming approximate factorization algorithm, a time marching implicit scheme that solves the Navier-Stokes equations in strong conservation form. It uses second order accurate central-differencing for spatial derivatives. The code was originally developed as AIR3D by Pulliam and Steger [19]; Pulliam [20] later added the Jameson [21] artificial dissipation and called the code ARC3D. Cooper [22] adapted the code for internal flows in propulsion applications and named the code PARC3D.

For the present application, the equations are solved for the steady laminar flow solution in the leading edge region, with the algebraic BaldwinLomax [23] turbulence model turned on just down- stream of the juncture between the cylinder and flat plate. During the initial part of the calculation, the turbulence is updated at each iteration; then it is frozen and only recalculated each time the calculation is restarted, roughly every 2500 iterations. This freezing of the turbulence conserves computer time, and enhances the rate of convergence.

The turbulence model is used here simply to enhance convergence by limiting any reverse flow which may occur in this region. The primary focus is the leading edge region, which should not be greatly affected by the turbulence downstream. Because of the central-differencing, the code has Jameson [21] type, fourth order, artificial dissipation for stability. The artificial dissipation is added both explicitly and implicitly; the coefficient varies both spatially and directionally to minimize the dissipation [24, 25]. Although a dissipation model which varied the coefficient spatially but not directionally was too dissipative, it gave very good convergence rates. Because of this, the localdirectional model was used both upstream of and on the cylindrical leading edge, and the local model was used both on the flat plate portion and downstream of the leading edge. In a problem of this type, it is especially important to use a method where the decay of freestream vorticity from numerical dissipation is small compared to the decay from the physics of the flow. Included in the results is a demonstration that the decay of freestream vorticity is properly calculated.

A C-grid, shown in Figure 2, was used; half of the grid was eliminated by imposing symmetry in the $x-z$ plane. The grid extended 10 radii up and downstream and ranged from $z=\lambda / 2$ to $z=\lambda$. 
The PARC3D code requires about 30 words of storage per grid point and $22 \times 10^{-6}$ seconds of CPU time per iteration per grid point on the NAS Cray Y/MP. Each case took approximately 5000 iterations, for typical run times on the order of $5000 \mathrm{CPU}$ seconds. For each value of $\lambda$, the problem was first solved for some value of $A_{0}$, then $A_{0}$ was changed, and the previous solution was used as an initial guess. This resulted in fewer iterations required for subsequent runs.

\section{Boundary Conditions}

A symmetry condition was imposed at $z=\lambda / 2$ and $z=\lambda$. Symmetry was imposed by using second order accurate one-sided differences.

On the body, no-slip, constant heat flux conditions were enforced, along with a zero normal pressure gradient. At the exit, static pressure was imposed while velocity components and total energy were linearly extrapolated. The upstream boundary condition is determined by specifying the total temperature and total pressure, and requiring that the flow be aligned with the $\mathrm{x}$-direction. Then at each itcration, the upstream running Riemann invariant is extrapolated to predict the velocity and density. The original code used zeroeth order extrapolation, evaluating the invariant one grid point in from the boundary and imposing it at the boundary. Using this technique resulted in a "kink" in the decay of vorticity at the upstream boundary; that is, the vorticity showed very little decay between the first two grid points and then decayed normally. This problem was greatly reduced by using a linear extrapolation for the Riemann invariant.

To produce a sinusoidal velocity variation at the upstream boundary, the total pressure was spccified by

$$
p_{t}=p_{e}\left\{1+\frac{\gamma-1}{2}\left[M^{\prime}\left(1-\frac{A_{0}^{\prime}}{2}\left(1+\cos \left(\frac{2 \pi z}{\lambda}\right)\right)\right]^{2}\right\}^{\frac{\gamma}{\gamma-1}}\right.
$$

where $M^{\prime}$ is approximately the peak Mach number at the upstream boundary and $A_{0}^{\prime}$ will be roughly the peak-to-peak velocity difference as a percentage of the inlet velocity. This boundary condition will produce a Mach number and amplitude slightly different from $M^{\prime}$ and $A_{0}^{\prime}$. The results are reported based on the actual solution, not on the idealized parameters. For all of the present cases $M^{\prime}=0.2$, resulting in freestream $\mathrm{Mach}$ numbers of roughly 0.17 . The total temperature was specified by

$$
T_{t}=T_{0}\left\{1+\frac{\gamma-1}{2}\left[M^{\prime}\left(1-\frac{A_{0}^{\prime}}{2}\left(1+\cos \left(\frac{2 \pi z}{\lambda}\right)\right)\right]^{2}\right\}\right.
$$

This total temperature profile was imposed simply as an aid to assess the numerical dissipation of the code.

This profile produces a uniform entropy profile at the upstream boundary. By running the code without the viscous terms, it was possible to see the effects of the numerical dissipation in terms of the production of entropy. The inviscid calculations were used to indicate the number of grid points required for an accurate calculation of the decay of vorticity in the freestream. The total temperature profile was left as stated above for all the runs, since its effect is negligible at the low Mach numbers.

\section{Decay of vorticity in the freestream}

To approximate the rate of decay of vorticity between the upstream boundary and the body, consider the flow of an incompressible fluid in the $x-z$ plane. Flow is assumed to be in the $x$-direction only, with a periodic variation in the $z$-direction. In addition, assume that the pressure gradient in the $x$-direction is zero and that the initial periodic variation is small compared to the average velocity. Then, let the velocity difference be

$$
u_{1}(x, z)=\bar{U}-u(x, z)
$$

where $\bar{U}$ is the average velocity. The appropriate equation governing the velocity difference, $u_{1}$, is

$$
\frac{\partial u_{1}}{\partial x}=\frac{\nu}{\bar{U}} \frac{\partial^{2} u_{1}}{\partial z^{2}}
$$

assuming $u_{1} / \bar{U}<<1$, where the boundary layer assumption has been made and terms quadratic in $u_{1}$ and $w_{1}$ have been neglected; $w_{1}$ is the cross stream velocity, which is being neglected. The boundary conditions are

$$
\begin{aligned}
& \text { at } x=x_{0} \quad: \quad u_{1}\left(x_{0}, z\right)=\frac{A_{0} \bar{U}}{2} \cos \left(\frac{2 \pi z}{\lambda}\right) \\
& \text { periodic : } u_{1}(x, 0)=u_{1}(x, \lambda)
\end{aligned}
$$

where $A_{0}$ is an arbitrary constant, but must be small compared to one. The solution to this equation with these boundary conditions is

$$
u_{1}(x, z)=\frac{A_{0} \bar{U}}{2} \cos \left(\frac{2 \pi z}{\lambda}\right) \exp \left(-\frac{4 \pi^{2}}{R e_{\lambda}} \frac{\left(x-x_{0}\right)}{\lambda}\right)
$$

where $R e_{\lambda}=\bar{U} \lambda / \nu$. Of particular interest is the vorticity, which is oriented out of the $x-z$ plane, and can be written as

$$
\begin{aligned}
\Omega(x, z) & =\frac{\partial u_{1}}{\partial z} \\
& =-\frac{\pi A_{0} \bar{U}}{\lambda} \sin \left(\frac{2 \pi z}{\lambda}\right) \exp \left(-\frac{4 \pi^{2}}{R e_{\lambda}} \frac{\left(x-x_{0}\right)}{\lambda}\right)
\end{aligned}
$$

Notice that the level of vorticity is cverywhere directly proportional to the constant $A_{0}$, but the rate of decay is strongly dependent on the wavelength. Increasing $\lambda$ 

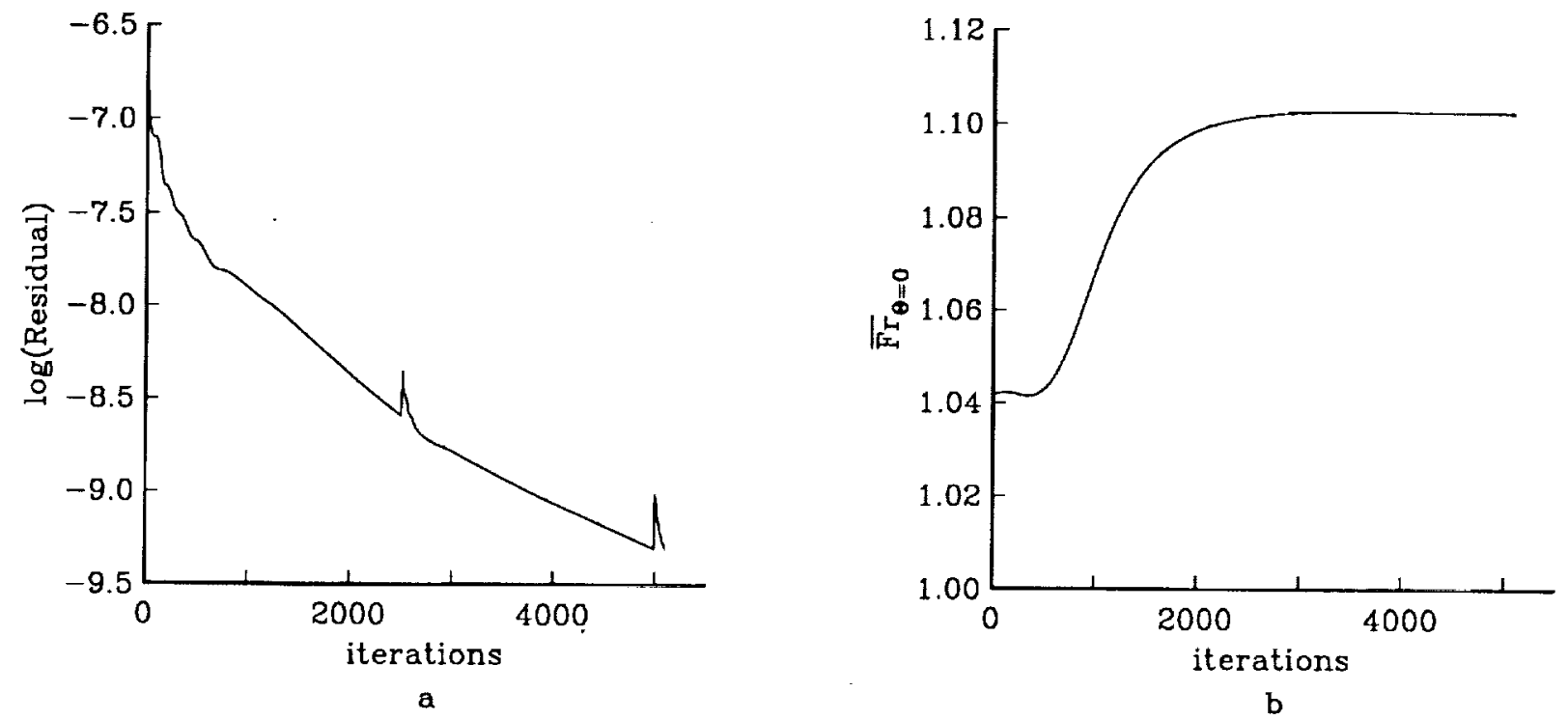

Figure 3: Convergence History for $\lambda=0.4 R, A=0.04, R e_{R}=6500$

decreases the initial value of vorticity at $x=x_{0}$, but it also decreases the rate of decay so that at some distance downstream, the level of vorticity will be greater than that for a case with a smaller $\lambda$ and the same $A_{0}$. The wavelength which results in the maximum peak vorticity at some distance downstream, to be referred to as the optimum wavelength $\lambda_{\text {opt }}$, can be written as

$$
\lambda_{o p t}=\Delta x \sqrt{\frac{8 \pi^{2}}{R e_{\Delta x}}}
$$

where $\Delta x=x-x_{0}$ and $\operatorname{Re}_{\Delta x}=\bar{U}\left(x-x_{0}\right) / \nu$. Note that the optimum wavelength is directly proportional to $\sqrt{\Delta x}$ for a given velocity, and inversely proportional to $\sqrt{\operatorname{Re}_{\Delta x}}$ for a given $\Delta x$.

The main result of this analysis is the finding that an upstream variation with the same amplitude but a longer wavelength will not necessarily create a smaller disturbance at the leading edge of the body. Further discussion of this point will appear below as it pertains directly to the numerical results.

\section{Results}

For the present study, the effect of varying the wavelength $\lambda$ and the amplitude $A_{0}$ of the upstream velocity profile are considered. The Reynolds number based on the leading edge radius is 6500 , and the fixed wall heat transfer is set at $q_{w}=4 k_{0} T_{0} / R$ for all cases, where $k_{0}$ and $T_{0}$ are the freestream thermal conductivity and temperature, respectively. This level of heat transfer results in a temperature rise of roughly $10 \%$ above the freestream absolute temperature. Calculations were run for three values of $\lambda$, $\lambda=0.2 R, 0.4 R$, and $0.8 R$. The parameter $A_{0}$ varied through the range 0.0 to 0.066 . The grid used for these cases was $37 \times 51 \times 22$, in the streamwise, normal, and spanwise directions, respectively. The case of $A_{0}=0$ was calculated using the same $x-y$ grid as the threc dimensional cases, but only five grid points in the $z$ direction. The case of $\lambda=0.2 R$ and $A_{0}=0.066$ was also calculated, using a grid of $76 \times 101 \times 42$ to check grid dependency. For the cases with $\lambda=0.2 R$, unjform grid spacing in the $z$-direction was used. After running the $\lambda=0.4 R$ cases, the decision was made to use geometric stretching in the spanwise direction to cluster points in the region where the vortex forms. The $\lambda=0.4 R$ cases were then rerun. Comparison of these two sets of runs is used as an additional check on grid dependency. The $\lambda=0.8 R$ cases were done with geometric stretching in the $z$-direction.

\section{Convergence Criterion}

For each case the code was run until the residual dropped below $5 \times 10^{-10}$, corresponding to a drop of roughly three orders of magnitude from the initial level. The residual is defined as the sum of the L2 norms for each of the five equations divided by the number of points. Figure 3 shows the history for the case of $\lambda=0.4 R$ and $A_{0}=0.04$. This calculation was initialized using the converged solution for $A_{0}=0.026$ so the residual starts out relatively low then, and drops 2.5 orders of magnitude to meet the convergence criterion, Figure 3a. The spikes in this figure result from the update of the turbulence each time the calculation is restarted. Recall that turbulence is only calculated on the flat plate portion of the body and is frozen after the initial drop in the residual. Notice 


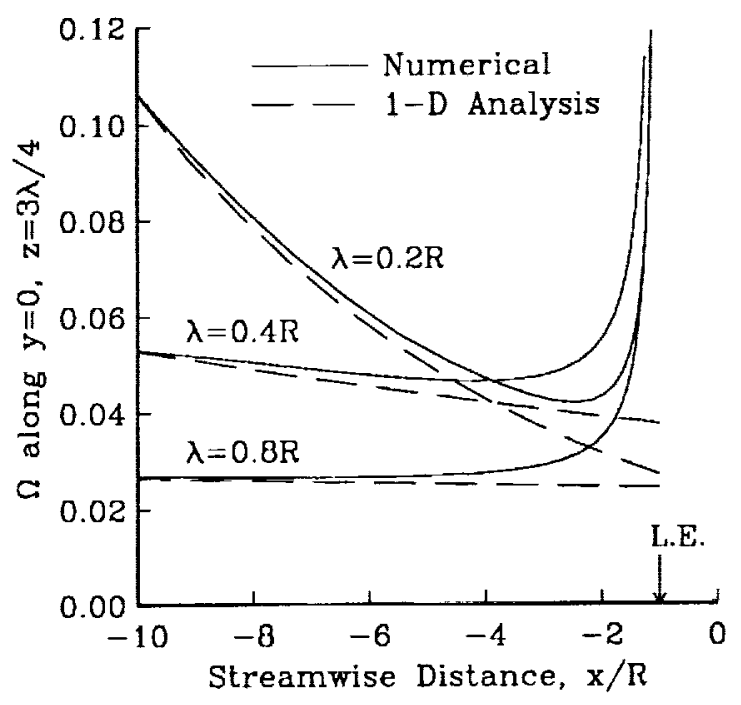

Figure 4: Decay of Peak Vorticity, $A_{0}=0.04, R e_{R}=$ 6500

that the spanwise-averaged Frössling number at the leading edge, Figure $3 \mathrm{~b}$, has settled long before the calculation is terminated. The Frössling number is defined as the Nusselt number divided by the square root of the Reynolds number. This figure is representative of the other cases run.

\section{Decay of Vorticity in the Freestream}

Figure 4 demonstrates the decay of vorticity in the streamwise direction and the amplification near the leading edge. Shown in this plot is the $y$ component of vorticity as a function of $x$ along the line $y=0, z=3 \lambda / 4$. For the same value of $A_{0}=0.04$, the $\lambda=0.2 R, 0.4 R$, and $0.8 R$ results are shown. Also shown in this plot are the analytical results from equation 9 with $A_{0}$ defined so that the vorticity corresponds to the numerical result at $x=x_{0}$. Recall that the analysis does not take into account the presence of the body, and thus does not predict the amplification near the leading edge. This plot demonstrates the importance of the rate of decay in determining the supply of vorticity to the leading edge region. Notice that the $\lambda=0.2 R$ case has the largest initial vorticity and yet does not result in the strongest vortex because of the high rate of decay. This plot also indicates that there is an optimum wavelength in terms of supplying the most vorticity to the leading edge for a given $A_{0}$.

The optimum wavelength for the present Reynolds number and upstream location from equation 10 is

$$
\lambda_{\text {opt }}=0.33 R
$$

which is in agreement with the observation, from the numerical computation, that $\lambda=0.4 R$ results in a stronger structure than the $\lambda=0.2 R$ or the $\lambda=0.8 R$

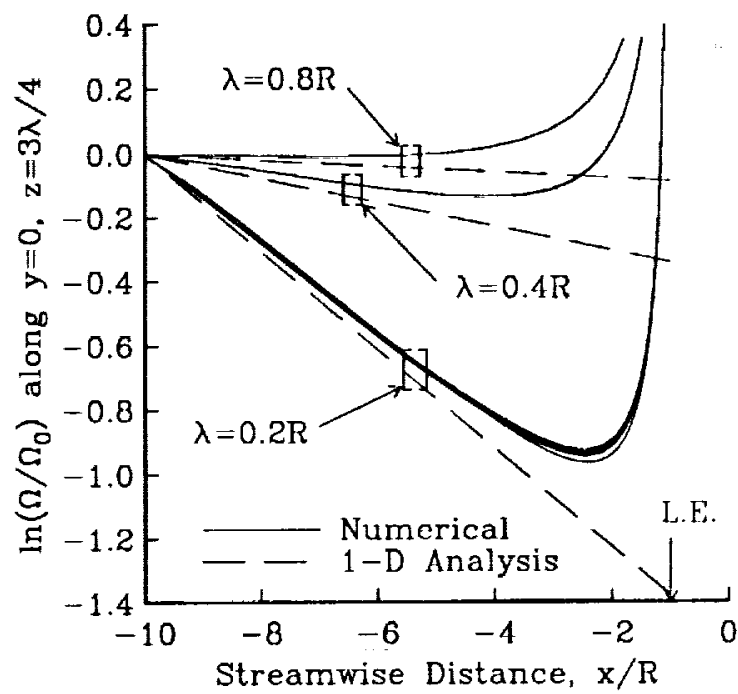

Figure 5: Decay of Normalized Peak Vorticity, $R c_{n}=$ 6500

cases. Notice from equation 10 that this optimum wavelength varies inversely with $\sqrt{R e_{\Delta x}}$. If $R e_{R}$ were varied while holding $R / \Delta x$ constant, then the previous statement would imply that the optimum wavelength varies as the inverse of $\sqrt{R e_{R}}$. It is very important to notice, however, that the optimum wavelength does not vary with $R$. (i.e., if $R e_{R}$ is varied by changing $R$, then the optimum wavelength would not vary). Various experiments $[26,27,28,29]$ with levels of turbulence on the order of 0.02 and less have observed spanwise variations with wavelengths that vary inversely as the square root of $R e_{R}$. This observation is often used to support the argument that the disturbance must be caused by the instability of the leading edge boundary layer. However, if the upstream variation is made up of many wavelengths, then the wavelength of the resulting disturbance could be seen to vary inversely with $\sqrt{R e_{R}}$, when the freestream velocity is varied, because the optimum wavelength predicted by the parallel flow analysis varies as $\sqrt{R e_{R}}$. It is not possible at this point to reach a conclusion, but it would appear that further investigation of the question of supply versus stability is warranted.

Figure 5 demonstrates further the agreement between the calculation and the analysis in the region of decaying vorticity. In this figure there are fifteen separate solid lines corresponding to all of the cases calculated; six cases with $\lambda=0.2 R$, six cases with $\lambda=0.4 R$, and three cases with $\lambda=0.8 R$. By plotting the vorticity normalized by its upstream value, the common rates of decay for each wavelength at several values of $A_{0}$ can be seen. Also shown in Figure 5 is the analytical result for each wavelength. The agreement is quite good considering the simplicity of the analysis. 

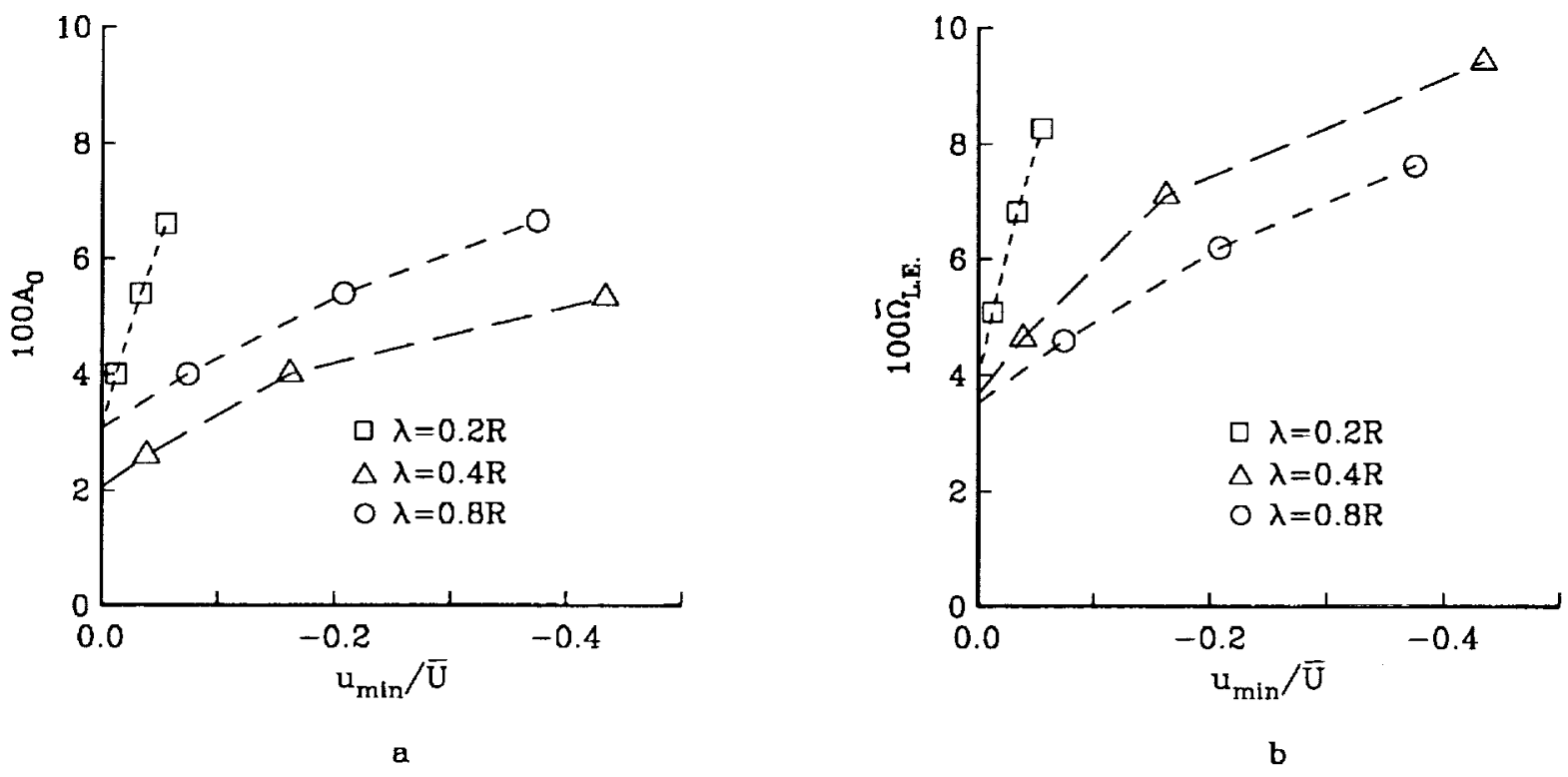

Figure 6: Extrapolation to Threshold Level

Once again, recall that the analysis does not take into account the presence of the body, and thus does not predict the amplification near the leading edge.

\section{Threshold Level for Vortex to Form}

It was observed numerically that a minimum amplitude at each wavelength is required for a vortex to form. A vortex is considered to have formed when reverse flow occurs in the $x-z$ symmetry plane. In Figure $6 \mathrm{a}$, the minimum streamwise velocity $u_{\min }$ is shown for three levels of $A_{0}$ for each wavelength. The levels of $A_{0}$ shown correspond to the first three values of $A_{0}$, which resulted in reverse flow for each wavelength. The predicted value of $A_{0}$ for $u_{\min }=0$ is then extrapolated by fitting a parabola thru the available data points. Figure $6 \mathrm{a}$ demonstrates that the minimum value for $A_{0}$ which generates a vortex structure differs by roughly $\pm 20 \%$ of the average for these three wavelengths. The peak level of vorticity supplied to the leading edge, as predicted by the parallel flow analysis, is evaluated for each case from

$$
\begin{aligned}
\tilde{\Omega}_{L . E .} & =\frac{\Omega(R, 3 \lambda / 4)}{\pi \bar{U} / R} \\
& =A_{0} \frac{R}{\lambda} \exp \left(-\frac{4 \pi^{2}}{R e_{R}}\left(\frac{R}{\lambda}\right)^{2} \frac{\left(R-x_{0}\right)}{R}\right)
\end{aligned}
$$

where $\left(R-x_{0}\right) / R=9$ for all of the present cases. This quantity is then extrapolated quadratically to the point of $u_{\min }=0$. The predicted level of vorticity to the leading edge differs by less than $\pm 5 \%$ for the three wavelengths considered in Figure $6 \mathrm{~b}$. From this result it seems possible that, for a given wavelength and Reynolds number, the minimum $A_{0}$ required for a structure to form could be estimated from

$$
A_{0, t h}=\frac{\lambda}{R} \tilde{\Omega}_{L . E, t h} \exp \left(\frac{4 \pi^{2}}{R e_{R}}\left(\frac{R}{\lambda}\right)^{2} \frac{\left(R-x_{0}\right)}{R}\right)
$$

where $\tilde{\Omega}_{L, E, t h}$ is a constant and is deduced from previous cases, and $\left(R-x_{0}\right)$ is the distance from the leading edge to the upstream boundary. It is very important to recall that the present analysis is only valid in regions where $A_{0}<<1$; thus, $\left(R-x_{0}\right)$ could not be interpreted as the distance to a screen upstream, although changes in $\left(R-x_{0}\right)$ would correspond directly to changes in the screen position. Notice that as the Reynolds number approaches infinity, the level of $A_{0}$ required at the upstream boundary decreases to a constant. Averaging the three extrapolated values gives $\Omega_{L . E \text {. th }}=0.037$. Using this value in equation 14 predicts the threshold level of $A_{0}$ for each wavelength to within $\pm 5 \%$ of the extrapolated values in Figure $6 a$.

It is interesting to note that if the vorticity upstream of the body occurs at the optimum wavelength as defined by equation 10 , then the threshold level for $A_{0}$ could be written as

$$
\left.A_{0, t h}\right|_{o p t}=\sqrt{\frac{\left(R-x_{0}\right)}{R} \frac{8 \pi^{2}}{R e_{R}}} \exp \left(\frac{1}{2}\right)
$$

This result implies that if vorticity is always supplied at the optimum wavelength, the threshold $A_{0}$ would vary inversely with $\sqrt{R e_{R}}$ and directly with $\sqrt{\left(R-x_{0}\right) / R}$.

Experimentally, the threshold level has been studied by Hodson and Nagib [3] and by Kottke [4]. In each case, a row of wires was placed upstream of a circular 
leading edge to produce a small spanwise variation in the upstream velocity. Kottke found that for Reynolds numbers such that the wire wakes were steady, the upstream position of the wires for impending vortex formation depended linearly on the Reynolds number based on the wire diameter and did not depend on the Reynolds number based on the leading edge diameter.

It is possible to explain this trend in terms of the supply of vorticity reaching the leading edge. If the position $x_{0}$ is considered to be a fixed distance downstream of the wires located at, say, $x_{w i r e}$, then

$$
R-x_{0}=R-x_{w i r e}-\left(x_{0}-x_{w i r e}\right)
$$

so changes in $\left(R-x_{0}\right)$ would correspond directly to changes in the distance from the wires to the leading edge. Requiring that the peak vorticity reaching the leading edge be a constant, equation 9 indicates that $\left(R-x_{0}\right)$ must vary linearly with $R e_{\lambda}$. This is equivalent to Kottke's observation, since the ratio of wire spacing to wire diameter is not changed in that experiment. This conclusion also assumes that the dominant wavelength and $A_{0}$ do not vary with $R e_{R}$. Hodson and Nagib [3] found a correlation between $\sqrt{R-x_{\text {wire }} / d}$ and $(1-\sigma) \sqrt{R e_{R}}$, where $\sigma$ is the solidity of the screen and $d$ is the wire diameter. They found a correlation for a single wire as well as for an array of wires with various wire diameters. The trend for each case agrees with the arguments of the present hypothesis. Ignoring the effects of solidity, this correlation is equivalent to a linear relation between $\left(R-x_{\text {wire }}\right)$ and $R e_{R}$. Since $R e_{R}$ was varied by changing the freestream velocity, $R e_{\lambda}$ will differ from $R e_{R}$ by only a multiplicative constant. In fact, if a set of data does exhibit this linear relation except for some scatter from error, then taking the square root of each quantity would tend to enhance the correlation.

\section{Heat Transfer Results}

Figure 7 shows the spanwise-averaged Frössling number $\overline{F r}$ as a function of $\theta$ for $A_{0}=0.04$ and $\lambda / R=0.2,0.4$, and 0.8 . Also shown are the analytical solution of Frössling [30] and the PARC3D calculation for $A_{0}=0$. The Frössling number is defined as

$$
F r=\frac{N u}{\sqrt{2 R e_{R}}}
$$

so

$$
F r=\frac{q_{w}}{k_{0} T_{t, 0}} \frac{2}{\sqrt{2 \operatorname{Re}_{R}}} \frac{k_{0}}{k_{f}} \sqrt{\frac{\mu_{f}}{\mu_{0}}}\left(\frac{T_{w}}{T_{t, 0}}-1\right)^{-1}
$$

where $N u$ is the Nusselt number, $q_{w}$ is the wall heat transfer, $T_{w}$ is the wall temperature, and $T_{t, 0}$ is the

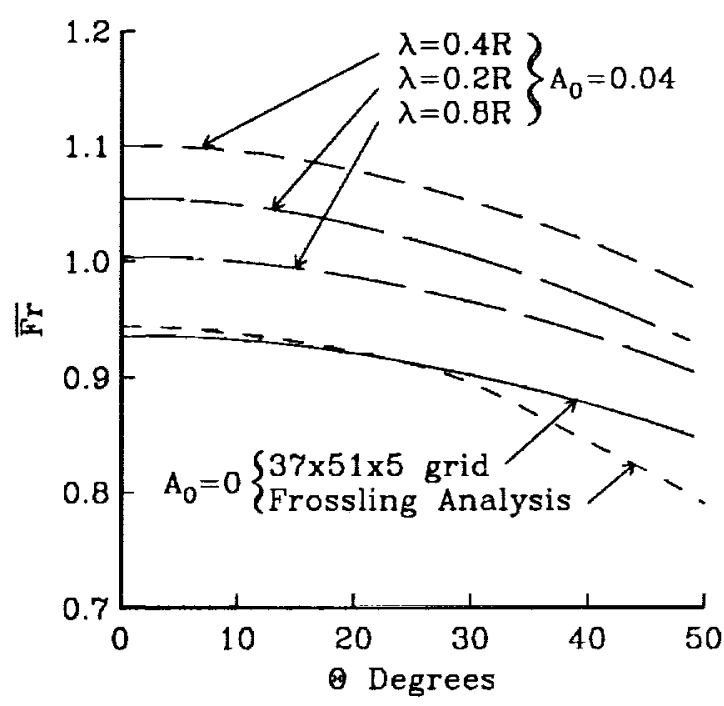

Figure 7: Frössling Number vs. Streamwise Distance, $A_{0}=0.04, R e_{R}=6500$

freestream total temperature. The thermal conductivity $k$ and viscosity $\mu$ are evaluated at the film temperature to minimize the effect of variable properties. Their effect for the present work is not large, but it should be noted that evaluating properties at the freestream temperature can result in Frössling numbers one to two percent higher than those presented. For the three-dimensional problem, the film temperature is defined by

$$
T_{f}=0.25\left(2 T_{t, 0}+T_{w, \max }+T_{w, \min }\right)
$$

Notice that the Frössling analysis and the twodimensional calculation (i.e., $A_{0}=0$ ) show good agreement for $\theta<30^{\circ}$. For $\theta>30^{\circ}$ the Frössling analysis decreases faster than the two-dimensional calculation. This decrease is indicative of a comparison between a constant wall temperature condition, which is what the Frössling analysis uses, and a constant wall heat flux condition [31]. Also notice in Figure 7, that for the same value of $A_{0}, \lambda / R=0.4$ causes the highest $\overline{F r}$, which is $18 \%$ higher at $\theta=0$ than the two-dimensional result from PARC3D. In fact, the spanwise-averaged Frössling number was higher than the two-dimensional result for every case considered. Increases in the transfer coefficient on the order of $15-20 \%$ have been seen experimentally $[2,3,6]$ in flows with upstream spanwise variation. Direct comparison with the present results would require knowledge of the wavelength and $A_{0}$ at several upstream locations so that an estimate of the vorticity supplied to the leading edge could be made.

Figure 8 shows the spanwise variation of Frössling number at $\theta=0$ for the same cases shown in Figure 7. Notice that locally, the Frössling number drops be- 


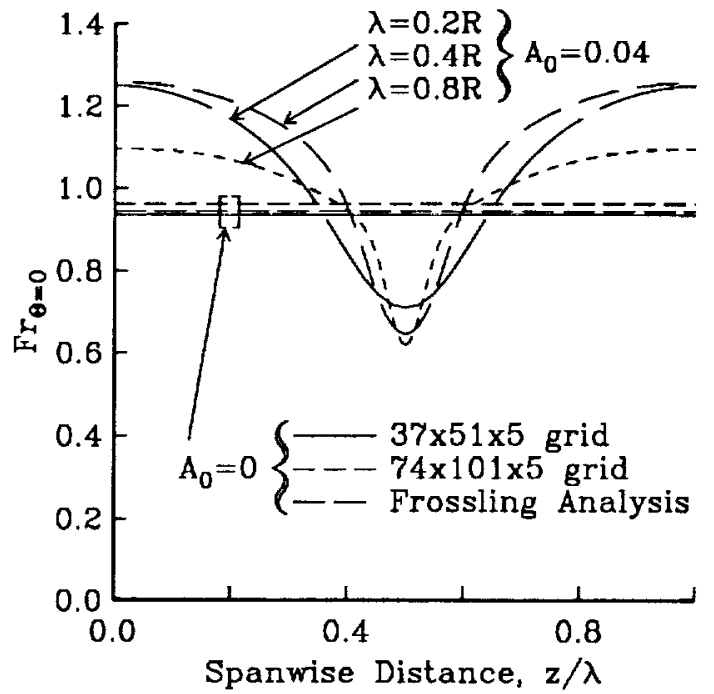

Figure 8: Frössling Number vs. Spanwise Distance, $A_{0}=0.04, R e_{R}=6500$

low the two-dimensional result. This reduction corresponds to the region directly in front of the lower speed flow and beneath the vortex pair. The drop in $F r$ is more than offset by the increase in $F r$ over most of the wavelength, causing the spanwise-averaged Frössling number to be higher than the two-dimensional result, as was shown in Figure 7. The implication of this observation is that the spanwise-averaged temperature will be lower, but locally the peak temperature will be greater than the two-dimensional value.

To demonstrate the grid dependence of the present calculation, Figures 9 and 10 show the Frössling number at the leading edge as a function of spanwise distance. Figure 9 shows the results for $\lambda=0.4 R$ and $A_{0}=0.04$ for two grids. The difference between the two grids is that one has geometric stretching in the spanwise direction, which clusters points in the region where the vortex forms, while the other grid has uniform spacing in the spanwise direction. The minimum spanwise spacing in the stretched grid is roughly a factor of 3 smaller than in the grid with uniform spacing in the spanwise direction. Notice that the Frössling number is only slightly affected by the change in grid. Figure 10 shows the results for $\lambda=0.2 R$ and $A_{0}=0.066$. The fine grid, which has twice as many grid points in each direction, falls within five percent of the normal grid calculation. It is expected that at lower values of $A_{0}$, the agreement would be even better.

As was mentioned previously, the spanwiseaveraged Frössling number was higher than the two dimensional result for every case considered. Figure 11 clearly demonstrates that a small spanwise variation in velocity is capable of causing a large increase

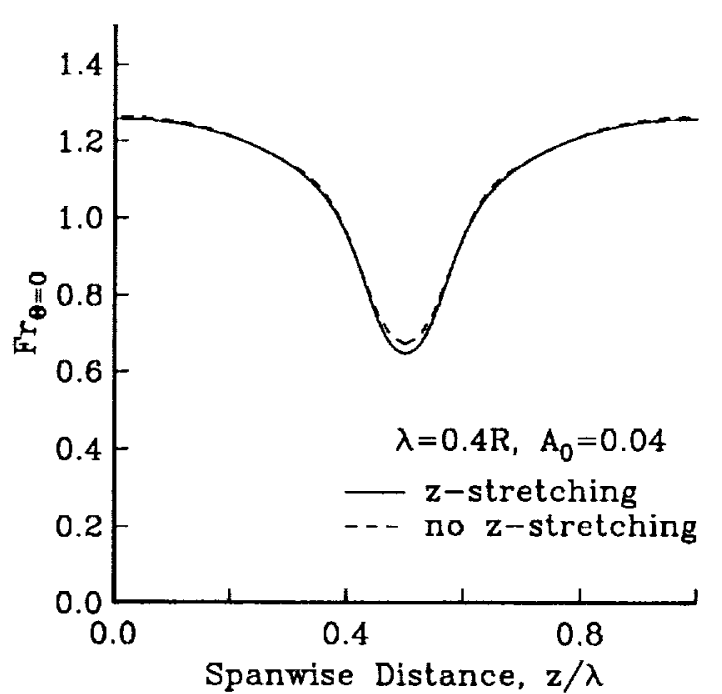

Figure 9: Comparison of Grid Dependence

in the heat transfer coefficient. It figure also demonstrates the minor effect that grid refinement has on the results.

\section{Conclusions}

From the present calculations it is hypothesised that a minimum level of vorticity must be supplied to the leading edge for a vortex to form. Using a simple parallel flow analysis to predict the decay of vorticity, it is further proposed that the appearance of a stationary vortex structure can be predicted from upstream conditions. If a disturbance, dominated by a single wavelength, is produced upstream of a leading edge, then the maximum distance between the disturbance and leading edge that will allow the formation of a stationary vortex structure will vary linearly with the Reynolds number based on the dominant wavelength. This statement is shown to agree qualitatively with experimental observations $[4,3]$ as well as with the present numerical solution of the Navier-Stokes equations.

The parallel flow analysis also indicates that an optimum wavelength exists for a given distance to the upstream disturbance and a given Reynolds number based on that distance. This optimum wavelength is found to vary as the inverse of $\sqrt{R e_{\Delta x}}$. This result may explain the appearance of spanwise variations in experiments, with wavelengths that vary inversely as the square root of the Reynolds number, as opposed to being explained by an instability in the stagnation flow as has been proposed in the past. If upstream of the leading edge, a spanwise variation exists consisting of many different wavelengths, then the wavelength supplying the most vorticity to the leading edge would vary as the inverse of $\sqrt{R e_{\Delta x}}$ from the above 


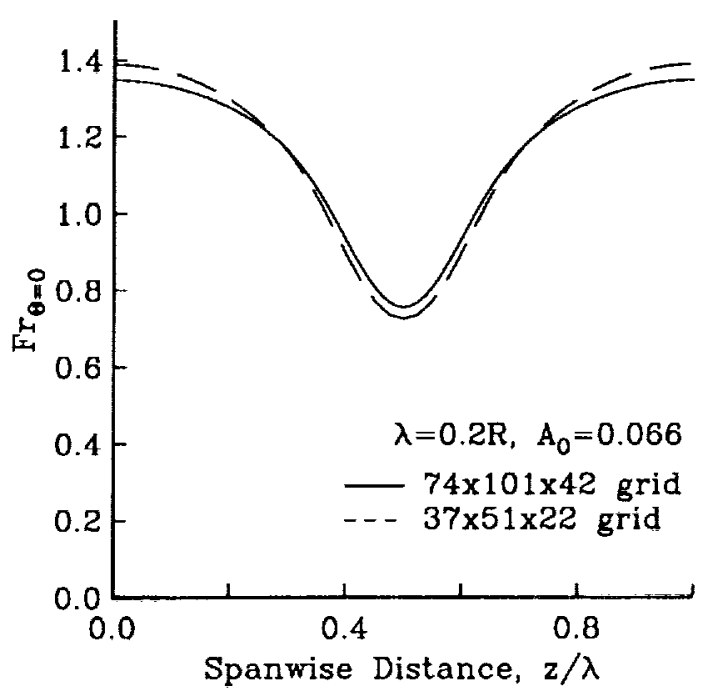

Figure 10: Comparison of Grid Dependence

argument. If $R e_{R}$ varies while holding the ratio $\Delta x / R$ fixed, this would imply a variation with $\sqrt{R e_{R}}$, which is what has been seen experimentally. It should be noted that even if the decay is not occurring exactly as the present analysis would suggest, the trends may still remain unchanged. For instance, Townsend [32] has indicated that the amplitude of a periodic disturbance in mean velocity for turbulent flow should decay as in equation 7 , except that the coefficient in the exponential would be increased by the ratio of the turbulent to kinematic viscosity. This technique would not contradict any of the above mentioned trends.

For all cases considered in this study, it was found that introducing a spanwise variation into the freestream always caused an increase in the spanwiseaveraged heat transfer coefficient. The percentage increase in the heat transfer coefficient was substantially larger than the freestream disturbance expressed as a percentage of the freestream velocity. For example, a 0.04 disturbance with wavelength $0.4 R$ located 9 radii upstream of the leading edge resulted in an increase in the heat transfer coefficient of $18 \%$ above the two dimensional result. This result demonstrates that a small spanwise variation upstream of a body can be an important factor in determining the heat transfer to that body.

\section{References}

[1] J. Kestin and R. T. Wood. Enhancement of stagnation-line heat transfer by turbulence. Progress in Heat Mass Transfer, 2:pp. 249-253, 1969.

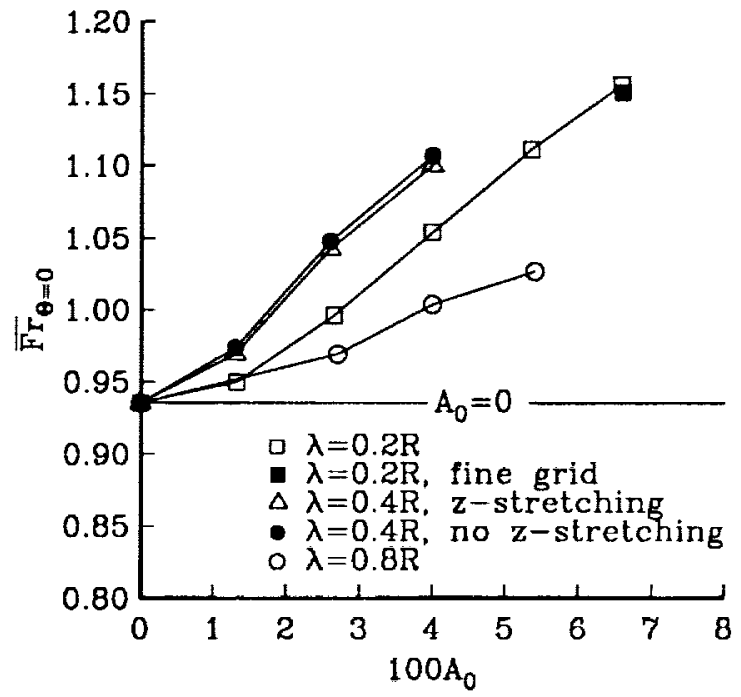

Figure 11: Frössling Number vs. $A_{0}, R e_{R}=6500$

[2] G. J. VanFossen Jr. and R. J. Simoneau. A study of the relationship between free-stream turbulence and stagnation region heat transfer. ASME J. Heat Transfer, 109:pp. 10-15, 1987.

[3] P. R. Hodson and H. M. Nagib. Vortices induced in a stagnation region by wakes: Their incipient formation and effects on heat transfer from cylinders. paper 77-790, AIAA, June 1977.

[4] V. Kottke. On the visualization of longitudinal vortices in stagnation flows. In Flow Visualization $I V$, pages 321-326. Hemisphere Publishing Corp., 1986.

[5] V. Kottke and B. Mpourdis. On the existence of Taylor-Görtler-vortices on concave walls. In Flow Visualization IV, pages 475-480. Hemisphere Publishing Corp., 1986.

[6] M. L. Marziale and R. E. Mayle. Mass transfer from a circular cylinder: Effects of flow unsteadiness and 'slight nonuniformities'. Technical Report CR-174759, NASA, Sept 1984. Rensselaer Polytechnic Institute, Troy, New York.

[7] J. Kestin and R. T. Wood. On the stability of two-dimensional stagnation flow. J. Fluid Mech., 44:pp. 461-479, 1970.

[8] G. Williams. Enhancement of heat and mass transfer in a stagnation region by free stream vorticity. paper 68-0022, ARL, February 1968.

[9] S. P. Sutera, P. F. Maeder, and J. Kestin. On the sensitivity of heat transfer in the stagnation point boundary layer to free-stream vorticity. J. Fluid Mech., 16:pp. 497-520, 1963. 
[10] S. P. Sutera and G. Williams. The effect of external vorticity on stagnation-point heat transfer at high Prandtl number. Int. J. Heat and Mass Transfer, 11:pp. 1795-1806, 1968.

[11] R. J. Swigart. Effects of vorticity amplification in two-dimensional and axisymmetric stagnationpoint flows. paper 77-92, AIAA, Jan 1977.

[12] G. Hämmerlin. On instability theory of plane stagnation flow. In H. Görtler and W. Tollmien, editors, Fifly Years of Boundary Layer Theory. Wiewig and Sohn, 1955. pp. 315-327.

[13] S.D.R. Wilson and I. Gladwell. The stability of a two-dimensional stagnation flow to threedimensional disturbances. J. Fluid Mech., 84, No. 3:pp. 517-527, 1978.

[14] C. Thompson and M. G. Manley. Linear stability of stagnation flow. J. Fluid Mech., 84, No. 3:pp. 517-527, 1978.

[15] M. V. Morkovin. On the question of instabilities upstream of cylindrical bodies. CR 3231, NASA, 1979.

[16] B. Sunden. A theoretical investigation of the effect of freestream turbulence on skin friction and heat transfer for a bluff body. Int. J. Heat and Mass Transfer, 22:pp. 1125-1135, 1979.

[17] R. M. Traci and D. C. Wilcox. Freestream turbulence effects on stagnation point heat transfer. AIAA Journal, 13:pp. 890-896, 1975.

[18] H. Miyazaki and E. M. Sparrow. Analysis of the free-stream turbulence on heat transfer and skin friction. ASME J. Heat Transfer, 99:pp. 614-619, 1977.

[19] T. H. Pulliam and J. L. Steger. Implicit finitedifference simulations of three dimensional compressible flow. AIAA Journal, 18:pp. 159-167, 1980.

[20] T. H. Pulliam. Euler and thin layer Navier-Stokes codes: ARC2D, ARC3D, March 1984. Notes for computational fluid dynamics user's workshop.

[21] A. Jameson, W. Schmidt, and E. Turkel. Numerical solutions of the Euler equations by finite volume method using Runge-Kutta time stepping schemes. paper 81-1259, AIAA, 1981.

[22] G. K. Cooper, J. L. Jordan, and W. J. Phares. Analysis tool for applications to ground testing of highly underexpanded nozzles. paper 87-2015, AIAA, 1987.
[23] B. S. Baldwin and H. Lomax. Thin layer approximation and algebraic model for separated turbulent flows. paper 78-257, AIAA, 1978.

[24] M. J. Siclari, P. DelGuidice, and A. Jameson. A multigrid finite volume method for solving the Euler and Navier-Stokes equations for high speed flows. paper 89-0283, AIAA, Jan 1989.

[25] G. Cooper and J. Sirbaugh. The PARC distinction: A practical flow simulator. paper 90-2002, AIAA, July 1990.

[26] J. Kestin. The effect of freestream turbulence on heat transfer rates. In T.F. Irvine and J.P. Hartnett, editors, Advances in Heat Transfer, volume 3. Academic Press, 1966.

[27] P. Colak Antič. Visual investigation of longitudinal vortices in the stagnation region of a circular cylinder exposed to a turbulent stream. Report on the DGLR-Specialist Session: "Laminar and Turbulent Boundary Layers," 1971. Göttingen, pp. 194-220.

[28] H. Hassler. Hot-wire measurements of occurrence of longitudinal vortex instability in the stagnation region of a circular cylinder. Report on the DGLR-Specialist Session: "Laminar and Turbulent Boundary Layers," 1971. Göttingen, pp. 221239.

[29] E.A. Brun, G.B. Diep, and J. Kestin. On a new type of longitudinal vortices in the flow around a cylinder. C.R. Acad. Sci. Paris, 263:pp. 742-745, 1966.

[30] N. Frössling. Evaporation, heat transfer, and velocity distribution in two-dimensional and rotationally symmetrical laminar boundary-layer flow. Technical Report TM-1432, NACA, 1940.

[31] A. Žukauskas and J. Žiugžda. Heat Transfer of a Cylinder in Crossflow. Hemisphere Publishing Corp., 1985. p. 98.

[32] A.A. Townsend. The Structure of Turbulent Shear Flow. Cambridge Univ. Press, 1956. pp. 56-63. 


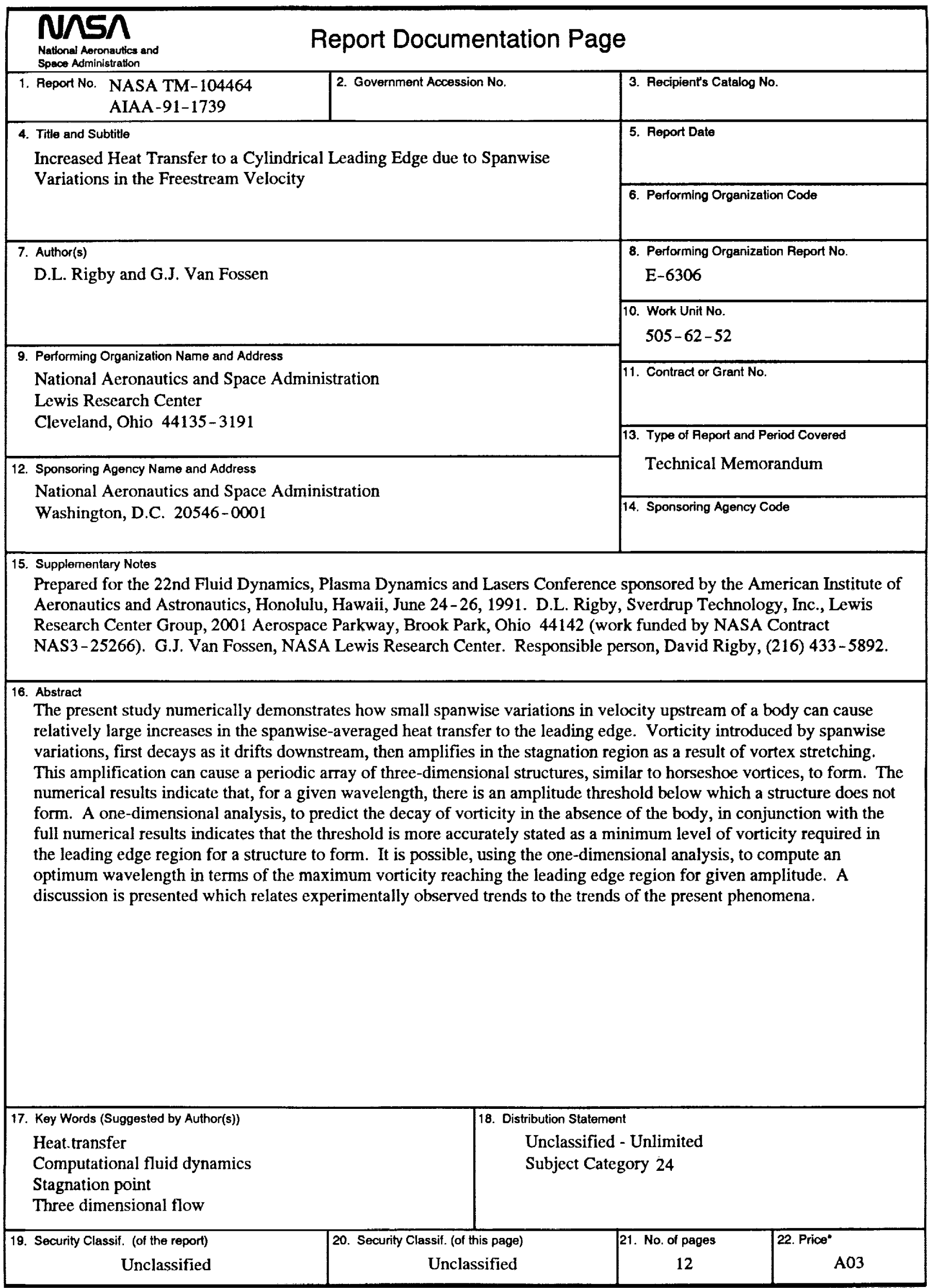

